Challenges in the extrapolation from DD to DT plasmas: experimental analysis and theory based predictions for JET-DT

This content has been downloaded from IOPscience. Please scroll down to see the full text.

2017 Plasma Phys. Control. Fusion 59014023

(http://iopscience.iop.org/0741-3335/59/1/014023)

View the table of contents for this issue, or go to the journal homepage for more

Download details:

IP Address: 84.88.53.148

This content was downloaded on $30 / 01 / 2017$ at $11: 48$

Please note that terms and conditions apply.

You may also be interested in:

Physics comparison and modelling of the JET and JT-60U core and edge: towards JT-60SA predictions

J. Garcia, N. Hayashi, B. Baiocchi et al.

Key impact of finite-beta and fast ions in core and edge tokamak regions for the transition to advanced scenarios

J. Garcia, C. Challis, J. Citrin et al.

Modelling of JET hybrid scenarios with GLF23 transport model: E $\times$ B shear stabilization of anomalous transport

I. Voitsekhovitch, P. Belo, J. Citrin et al.

Modelling of hybrid scenario: from present-day experiments towards ITER

X. Litaudon, I. Voitsekhovitch, J.F. Artaud et al.

Gyrokinetic study of turbulence suppression in a JET-ILW power scan

H Doerk, C Challis, J Citrin et al.

Chapter 6: Steady state operation

C. Gormezano, A.C.C. Sips, T.C. Luce et al.

On the different physical mechanisms for accessing hybrid scenarios on JET

J. Garcia, G. Giruzzi and JET EFDA Contributors

2008 Public Release of the ITPA Confinement Profile Database

C.M. Roach, M. Walters, R.V. Budny et al.

Improved confinement in JET high plasmas with an ITER-like wall

C.D. Challis, J. Garcia, M. Beurskens et al. 


\title{
Challenges in the extrapolation from DD to DT plasmas: experimental analysis and theory based predictions for JET-DT
}

\author{
J Garcia ${ }^{1}$, C Challis ${ }^{2}$, D Gallart ${ }^{3}$, L Garzotti' ${ }^{2}$, T Görler ${ }^{4}$, D King ${ }^{2}$, \\ M Mantsinen ${ }^{3,5}$ and JET contributors ${ }^{6}$ \\ EUROfusion Consortium, JET, Culham Science Centre, Abingdon, OX14 3DB, UK \\ ${ }^{1}$ CEA, IRFM, F-13108 Saint-Paul-lez-Durance, France \\ ${ }^{2}$ CCFE, Culham Science Centre, Abingdon, OX14 3DB, UK \\ 3 Barcelona Supercomputing Center (BSC-CNS), C. Gran Capità 2-4, 08034 Barcelona, Spain \\ ${ }^{4}$ Max Planck Institute for Plasma Physics, Boltzmannstr. 2, 85748 Garching, Germany \\ ${ }^{5}$ ICREA, Pg. Lluís Companys 23, 08010 Barcelona, Spain \\ E-mail: jeronimo.garcia@cea.fr
}

Received 1 July 2016, revised 26 August 2016

Accepted for publication 13 September 2016

Published 27 October 2016

\begin{abstract}
A strong modelling program has been started in support of the future JET-DT campaign with the aim of guiding experiments in deuterium (D) towards maximizing fusion energy production in Deuterium-Tritium (DT). Some of the key elements have been identified by using several of the most updated and sophisticated models for predicting heat and particle transport, pedestal pressure and heating sources in an integrated modelling framework. For the high beta and low gas operational regime, the density plays a critical role and a trend towards higher fusion power is obtained at lower densities. Additionally, turbulence stabilization by $E \times B$ flow shear is shown to generate an isotope effect leading to higher confinement for DT than DD and therefore plasmas with high torque are suitable for maximizing fusion performance. Future JET campaigns will benefit from this modelling activity by defining clear priorities on their scientific program.
\end{abstract}

Keywords: plasma, tokamak, integrated modeling

(Some figures may appear in colour only in the online journal)

\section{Introduction}

As part of its mission to prepare the operation of ITER, JET has undertaken numerous improvement and upgrades such as the replacement, among others, of the plasma face components (PFC) from Carbon wall by the iter like wall (ILW) with a mixture of Beryllium and Tungsten and the upgrade of the neutral beam injection (NBI) power. In order to further minimize risks of the future ITER Deuterium-Tritium (DT) operation, a second DT campaign, DTE2, has been as well envisaged for JET [1]. With this campaign, specific DT burning plasmas physics, as the fusion born fast ion generation, its interaction with turbulence, MHD or heating systems

${ }^{6}$ See the appendix of [24]. as well as the turbulence and pedestal behavior in multi-ion species plasmas could be properly studied.

In this framework, the predictability of such plasmas is a key issue for preparing DT experiments and designing and building credible future fusion devices. The integration of several physics aspects is mandatory for an accurate extrapolation from present day plasmas, mainly obtained with $\mathrm{D}$ as the main ion species, to conditions in which the ion mixture will be dominated by DT. This is an important challenge due to the scarceness of experimental data in support of the extrapolation efforts. In fact, some of the experimental data included in the present analysis belongs to the previous JET-DT campaign, DTE1 [2]. Their analysis is challenging given the fact that the data quality is not comparable to present-day standards [3]. Therefore, in general a combination of integrated 
modelling and first principle analyses is required to address the physics of burning plasmas. As an example, plasma turbulence in presence of different hydrogen isotopes and very energetic ions requires dedicated gyrokinetic analyses as the reduced models available now have been mainly tested in D plasmas with a fast ion component mainly obtained from the NBI heating. Additionally, the interplay between fusion born alpha particles and sawteeth requires a specific study.

Following this general guidelines, an extensive exercise of physics analysis, benchmark and integrated modeling has been undertaken in the framework of the future JET-DT campaign with the aim of investigating about the key physics of DT plasmas extrapolation, providing useful guidelines for establishing priorities in a potential DT campaign and establishing optimum operational regimes for maximizing the fusion power generation. Some of the essential physics analyses and integrated modelling activity to be performed in D and predicted DT plasmas to ensure the correct extrapolation and quantification of the expected key physics in DT [4] are the following:

1. Validate modelling codes on existing data from recent D campaigns via integrated-scenario modelling.

2. Identify and model ion resonant cyclotron heating (ICRH) schemes suited for maximizing fusion performance through core ion heating.

3. Predict DT performance, ICRH heating and ion acceleration efficiency, alpha particle effects, isotope effects and MHD stability in DT plasmas.

The modelling activity shown in this paper carefully follows the previous program and aims to provide input and guidance for scenario developers based on validated modelling. Additionally, it can provide a framework for establishing a general procedure for extrapolating JET plasmas to ITER DT regimes. We concentrate our effort on the validation with the most sophisticated models available of heat and particle transport, heat sources, pedestal pressure, optimum ICRH schemes, DT fusion power predictions and isotope effects. For that purpose, high beta plasmas at high power are considered due to their low power degradation [5]. The latter is beneficial for maximizing the fusion energy produced at high power, and thus, possibly allowing for a higher fusion gain power than expected from usual scaling laws. Nevertheless, high current plasmas with lower values of beta will be also investigated in the future to see if the favorable confinement scaling with plasma current can be exploited for high fusion performance.

A series of representative discharges from previous campaigns has been chosen for testing models and predictive capabilities. This is particularly important for first-principle models, as they can provide guidance in the cases where experimental data is limited.

The paper is organized as follows. In section 2 the general methodology applied for the extrapolation is shown and justified. In section 3, the codes and models applied are described. In section 4, integrated modelling is validated with DD plasmas. DT extrapolations are shown in section 5 whereas the impact of ICRH schemes, isotope effect and alpha heating and DT fueling is shown in section 6. Perspectives and conclusions will be addressed in section 7 .

\section{Extrapolation methodology}

The extrapolation of present day plasmas to DT requires validation of different modelling at several complexity levels and the extrapolation to some operational regimes which have not been attained yet at JET. One example is the hybrid scenario at high toroidal current $\left(I_{\mathrm{p}}\right)$, for which previous analyses have shown that high input power and large enough fast ion fraction are required in order to obtain the improved thermal energy confinement time usually obtained at low current [6]. Therefore, the verification of a suite of models in some particular (usually narrow) plasma condition is not enough for ensuring the correct prediction to DT, it is also needed to demonstrate that that choice is able to properly scale the plasma to at least different input power, $I_{\mathrm{p}}$ and toroidal magnetic field $\left(B_{\mathrm{t}}\right)$. For that purpose, the following strategy has been adopted in this study:

- Validation of models on existing D plasmas.

- Verification of a minimum extrapolation capability with existing D plasmas when changing power, $I_{\mathrm{p}}$ and $B_{\mathrm{t}}$.

- Verification of the extrapolation strategy with future D plasmas.

- Close the 'gap' with respect to DT physics: Validation of models with DTE1 and future isotope experiments.

- First-principle modelling supporting the extrapolation strategy.

Additionally, the extrapolation strategy to DT must reproduce key physics recently found at JET in D, such as deviation from the scaling IPB98 $(y, 2)$ [7] obtained in dedicated power scans both at low and high triangularity in low gas regimes. Some of the key physics that explain such deviation are the increased impact with power of several core plasma turbulence stabilizing mechanisms, such as the stabilization by electromagnetic effects and fast ions pressure gradients or $E \times B$ flow shear [8, 9]. Additionally, the increased beta tends to stabilize edge ballooning modes and expand the peelingballooning stability boundary leading to an increase of the pedestal pressure and the onset of a core-edge feedback loop through plasma stiffness. This is because they do not drive any core turbulence when the pressure gradients increase with power as long as the plasma remains in the ITG regime [9]. Therefore, these contributions are essential for a correct extrapolation to DT plasmas, which will self-generate extra heating power and a very energetic fast ion content due to fusion-born alpha particles.

\section{Codes and models applied}

Several integrated modelling suites have been used in these studies. One of them is the CRONOS suite of codes [10] which can solve the transport equations for various plasma fluid quantities (current, energy, particles, momentum). This is done in one dimension (the toroidal magnetic flux coordinate), self-consistently with 2D magnetic equilibrium which is calculated by means of the HELENA code [11]. The sources are computed by external modules coupled with the main 
Table 1. Main characteristics of the discharges analyzed in this paper.

\begin{tabular}{lllllllll}
\hline Shot & $I_{\mathrm{p}}(\mathrm{MA})$ & $B_{\mathrm{t}}(\mathrm{T})$ & $q_{95}$ & $\kappa / \delta$ & $\beta_{\mathrm{N}} / \beta_{\mathrm{N}, \mathrm{th}}$ & $H_{98}(y, 2)$ & $P_{\text {tot }}(\mathrm{MW})$ & $R_{\mathrm{NT}}\left(\mathrm{s}^{-1}\right)$ \\
\hline 84792 & 1.4 & 1.7 & 4.4 & $1.63 / 0.27$ & $2.85 / 2.45$ & 1.20 & 13 & $3.79 \times 10^{15}$ \\
84798 & 1.4 & 1.7 & 3.9 & $1.63 / 0.25$ & $1.43 / 1.30$ & 0.93 & 6.0 & $7.87 \times 10^{14}$ \\
86614 & 2.5 & 2.9 & 4.3 & $1.80 / 0.40$ & $2.30 / 2.00$ & 1.10 & 27 & $2.19 \times 10^{16}$ \\
\hline
\end{tabular}

Note: $I_{\mathrm{p}}$ is the total current, $B_{\mathrm{t}}$ the toroidal magnetic field, $\kappa$ elongation, $\delta$ triangularity, $\beta_{\mathrm{N}}=\beta \mathrm{a} B / I_{\mathrm{p}}$ normalized beta (with a the plasma minor radius), $\beta_{\mathrm{N}, \text { th }}$ normalized thermal beta, $H_{98}(y, 2)$ thermal confinement factor, $P_{\text {tot }}$ injected power and $R_{\mathrm{NT}}$ the measured neutron yield.
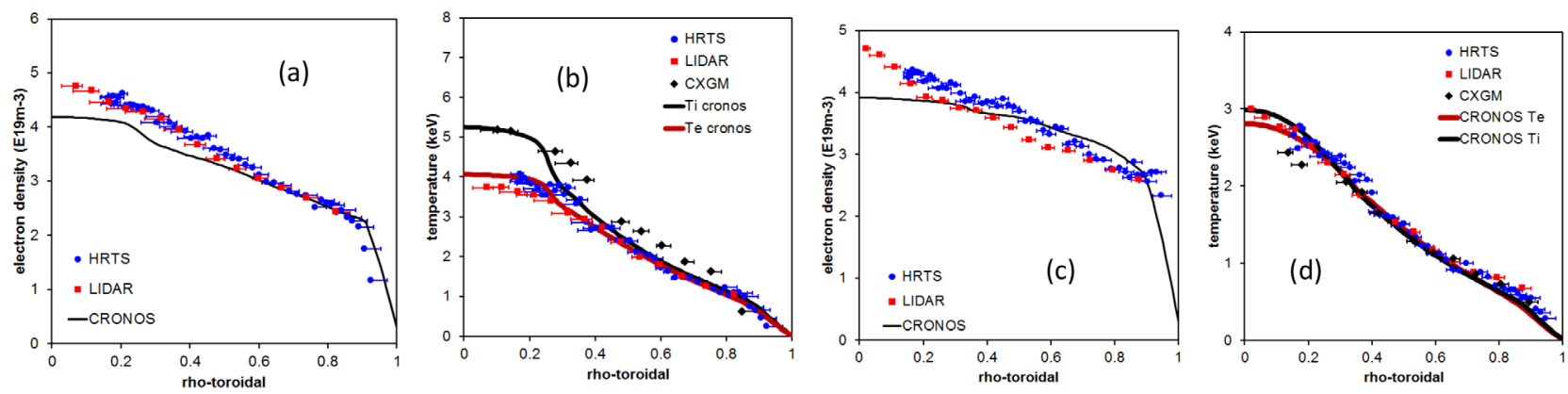

Figure 1. Comparison between the electron density and the electron and ion temperature profiles obtained with TGLF transport model and experimental data for the discharge 84792 ((a) and (b)) and 84798 ((c) and (d)). Horizontal error bars illustrate the uncertainty in the mapping from instrument line of sight to rho-toroidal.

transport equations. The neutral beam heating and current drive (NBH and NBCD) and the alpha power are computed by means of the NEMO/SPOT code [12]. The Ion Cyclotron Heating source is computed by the PION code [13], a time dependent 1D Fokker Planck code, including collisions and fast ion orbit losses. The transport model TGLF [14] is used for predicting core heat and particle fluxes whereas ion neoclassical transport is assumed for particle transport in the pedestal region.

In order to calculate the pedestal temperature, the following scaling [15] is used

$$
W_{\text {ped }}=0.000643 I^{1.58} R^{1.08} P^{0.42} n^{-0.08} B^{0.06} \kappa_{a}^{1.81} \varepsilon^{-2.13} m^{0.2} F_{q}^{2.09}
$$

where $I$ is the current (MA), $R$ major radius $(\mathrm{m}), P$ thermal loss power (MW), $n$ density $\left(10^{-19} \mathrm{~m}^{-3}\right), B$ toroidal field (T), $\kappa_{a}$ elongation, $\varepsilon$ aspect ratio, $m$ atomic mass and $F_{q}\left(\equiv q_{95} / q_{\text {cyl }}\right.$ with $q_{\text {cyl }}$ defined as $5 \kappa_{a} a^{2} B / R I$ with a minor radius). The position of the top of pedestal is fixed to the experimental value. This scaling has been shown to reasonably reproduce low delta C-wall hybrid discharges [16]. Additionally, its power dependence for the pedestal energy closely follows the one obtained in the recent power scan.

\section{Integrated modelling and extrapolation strategy validation}

Three discharges are selected to validate the integrated modelling and the extrapolation methodology. As representative of the low power degradation obtained in low triangularity $(\delta)$ and low gas ILW plasmas, the discharges 84792 and 84798 at $13 \mathrm{MW}$ and $6 \mathrm{MW}$ respectively are selected. Details of these discharges are shown in table 1 . The stabilizing effects due to $E \times B$ flow shear (the experimental toroidal rotation is used), electromagnetic fluctuations and the fast ion content as an extra species are considered. The characteristics of the fast ion content are taken from slowing-down distribution function. The gas level is adjusted in order to match the experimental density at the top of the pedestal when available.

The predictive simulation results are shown in figure 1 for discharge 84792 and they are compared to averaged measurements in the time window 5.2-5.5 s obtained by means of the high resolution thomson scattering (HRTS) and LIDAR for the electron and density temperature profiles and with charge exchange $(\mathrm{CX})$ for the ion temperature profile. HRTS density was re-normalized to match the interferometer density during the high beta phase of each pulse. In general the agreement between experimental data and simulation is acceptable in spite of the fact of a slightly under prediction of density peaking and temperatures at the top of the pedestal. In this context, one convenient parameter of merit used to evaluate the goodness of particular modelling is the measured DD neutron rate $\left(R_{\mathrm{NT}}\right)$. For the simulation performed here, the neutron rate is calculated with the code JETFUSE which estimates beamtarget and thermal fusion reactions based on a simple model using cross-sections from [17] and which has been validated in specific cases with NUBEAM/TRANSP [18] leading to fair agreement. The value obtained for the simulation of 84792 , $R_{\mathrm{NT}}=3.28 \times 10^{15} \mathrm{~s}^{-1}$ is $13 \%$ lower than the measured one.

As a part of the extrapolation strategy, a simulation has been performed by reducing the power to $6 \mathrm{MW}$ and comparing the results to the discharge 84798 from the power scan. The gas level is readjusted to match the pedestal density. The profiles obtained, shown in figure 1, although still showing a slight under prediction of density peaking and temperatures at the top of the pedestal are close to experimental data and $R_{\mathrm{NT}}=9.29 \times 10^{14} \mathrm{~s}^{-1}, 17 \%$ higher than the experimental measurement.

An additional step has been taken in order to consolidate the extrapolation strategy. The current is increased to 2.5 MA, 

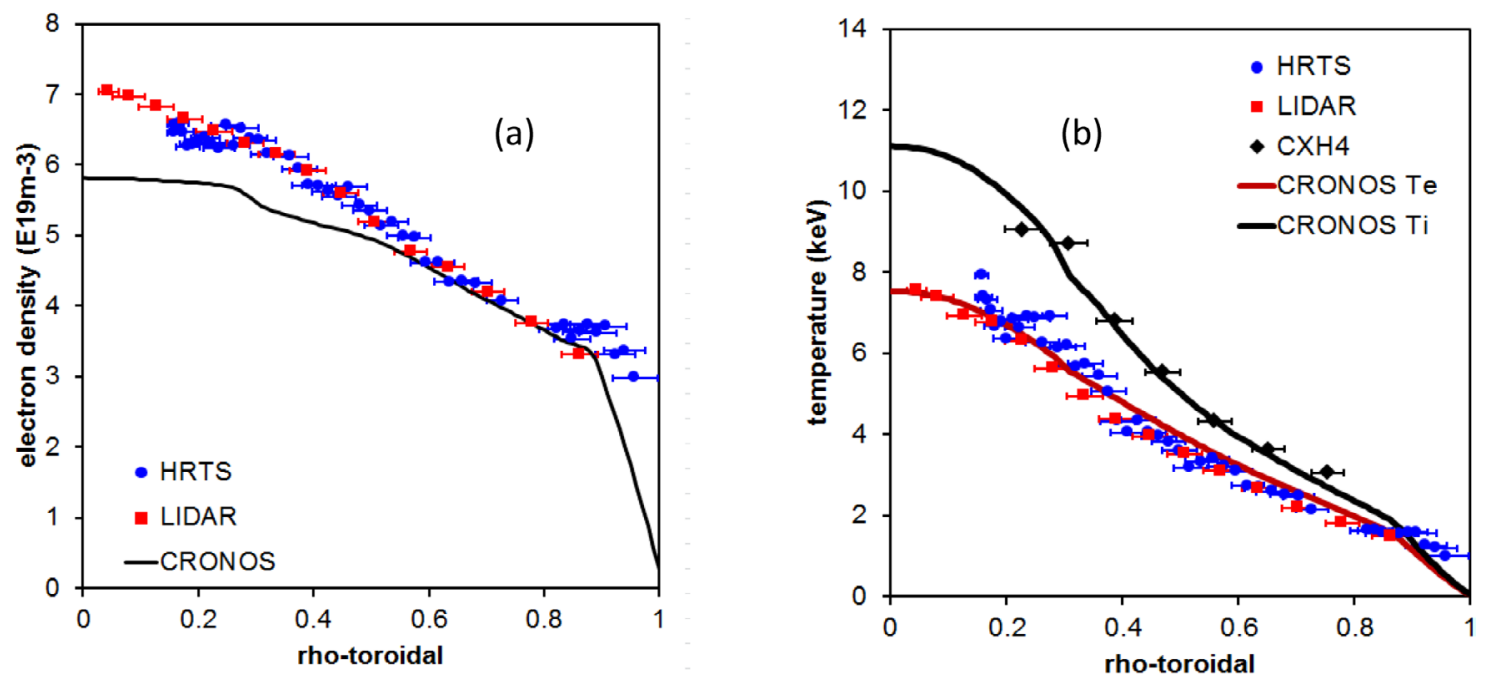

Figure 2. Comparison between the electron density (a) and the electron and ion temperature (b) profiles obtained with TGLF transport model and experimental data for the discharge 86614. Horizontal error bars illustrate the uncertainty in the mapping from instrument line of sight to rho-toroidal.
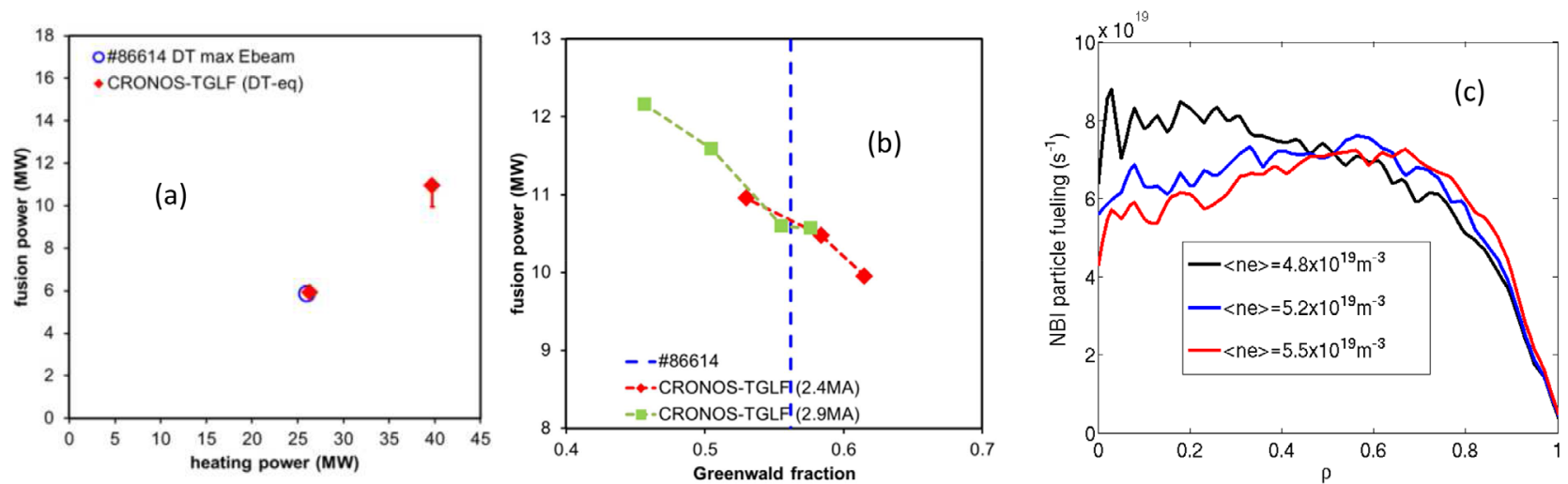

Figure 3. Equivalent fusion power calculation assuming 50\%D-50\% T mixture at different input power (a) sensitivity analysis of the fusion power dependence on the density with total input power of $40 \mathrm{MW}$ (b) NBI fuelling sensitivity to the average electron density (c).

the magnetic field to $2.9 \mathrm{~T}$ and the NBI power to $22 \mathrm{MW}$. Moreover, $5 \mathrm{MW}$ of ICRH power are added with hydrogen minority scheme at frequency $f=42.5 \mathrm{MHz}$. The results are compared to the discharge 88614 , with the same configuration, in the time window 8.2-8.5 s. This discharge holds the neutron yield record in the recent JET-ILW campaigns. As shown in figure 2 , the results are in reasonable agreement with experimental data with, again, a slightly density peaking underestimation. The neutron rate calculation, $R_{\mathrm{NT}}=1.76 \times 10^{16}$ $\mathrm{s}^{-1}$, is $19 \%$ lower than the measured one. Here, it should be noted, however, that the JETFUSE calculation does not take into account the ICRH acceleration of the NBI fast ions beam which can lead to an increase of $R_{\mathrm{NT}}$ of about $10 \%$ [19].

\section{DT extrapolation at full JET-ILW power}

The maximum fusion power that could be obtained at JET, following the operational domain previously described, is calculated based on the previous simulation with $P_{\mathrm{NBI}}=22 \mathrm{MW}$ by increasing $P_{\mathrm{NBI}}$ to the maximum power available, $34 \mathrm{MW}$. In these new simulations the gas levels and toroidal rotation profile are not changed with the increasing power. The equivalent fusion power is calculated with the JETFUSE code assuming a DT mixture of 50\%-50\% and including both the thermal and beam-target reactions. The uncertainties on the density top pedestal are analyzed by performing a scan on this parameter. Here, no credit for isotope effects of self-consistent alpha power generation and heating is considered.

As shown in figure 3 , the equivalent fusion power for $P_{\mathrm{NBI}}=22 \mathrm{MW}, P_{\text {fus }}=5.12 \mathrm{MW}$, is in fair agreement with the one obtained from interpretative simulations with TRANSP of the discharge $86614, P_{\text {fus }}=5.07 \mathrm{MW}$. When increasing the total power to $40 \mathrm{MW}$, then $P_{\text {fus }} \sim 11 \mathrm{MW}$, with $P_{\text {fus, thermal }}=3.45 \mathrm{MW}$ and $P_{\text {fus,beam }}=7.51 \mathrm{MW}$. However, this power is highly dependent on the density. In particular, we find that $P_{\text {fus }}$ decreases with increasing line average density, which means that in fact there is an optimum operational point in terms of density. The strong density dependence is confirmed by another scan at increased $I_{\mathrm{p}}=2.9 \mathrm{MA}$ and $B_{\mathrm{t}}=3.45 \mathrm{~T}$. At JET, the $I_{\mathrm{p}}$ increasing usually means a natural increasing of average density keeping the Greenwald fraction constant, something recovered in these simulations. At $I_{\mathrm{p}}=2.9 \mathrm{MA}$, the possible improvement of higher current is highly counteracted by the increased density, showing that density control is essential to 

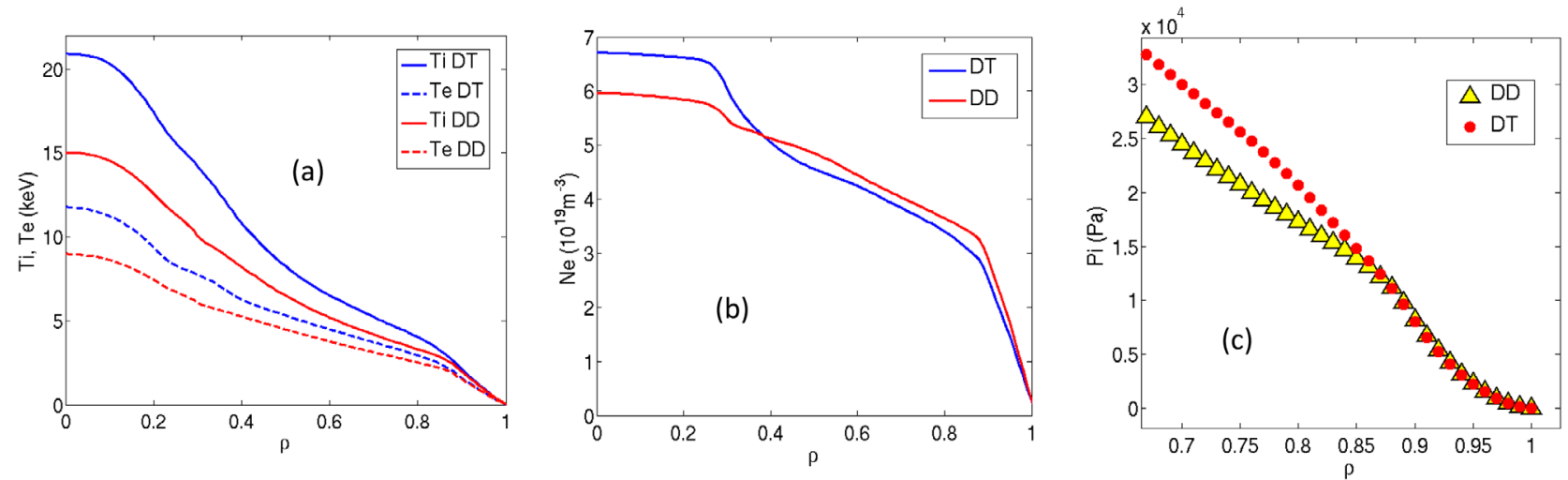

Figure 4. Comparison between the electron and ion temperatures and electron density profiles obtained with TGLF transport model for DD and DT mixtures ((a) and (b)) thermal ion pressure comparison for DD and DT (c).

maximize fusion power in this operational domain. In order to analyze by which physical mechanism the density has such a strong impact on the performance, the NBI particle fueling is shown in figure 3 for the different simulations. Clearly, the increased density involves a poorer beam penetration and a lack of inner core heating and fueling, which leads to poorer conditions for fusion power generation. Therefore central heating, in particular ion heating, becomes a necessity for avoiding loss of NBI heating when the density deviates from its optimum value. Several studies performed with the JETTO code and PION have shown that ${ }^{3} \mathrm{He}$ ICRH schemes can effectively improve ion core heating with respect $\mathrm{H}$ schemes [20]. This possibility will indeed be envisaged for a future DT campaign.

\section{Isotope and alpha heating effects}

The impact on turbulence and, consequently, on fusion power of the isotope change from DD to DT has been explored by performing simulations at maximum power and including $\mathrm{D}$ and $\mathrm{T}$ species in TGLF by assuming a 50\% D-50\% T mixture. Both species are assumed to have the same characteristics except the mass. As a first step, and in order to have a clear comparison of the impact of just heat and particle transport effects, all the sources are preserved in DT from the extrapolation performed with DD at $I_{\mathrm{p}}=2.9 \mathrm{MA}$ and $B_{\mathrm{t}}=3.45 \mathrm{~T}$ leading to $P_{\text {fus }}=10.94 \mathrm{MW}$. In figure 4 , the resulting electron and ion temperatures as well as the electron density are shown. Both ion and electron temperatures show a significant increase from DD to DT, especially strong for the ion channel with an increasing of the ion temperature peaking in the inner core region. This is due to a stronger turbulence stabilization of core turbulence in DT than in DD, which also leads to an increase of density peaking for DT specially pronounced as well at rho $<0.5$. Therefore, the equivalent fusion power also significantly increases in DT, $P_{\text {fus }}=16.34 \mathrm{MW}$ due to the isotope effect. Interestingly, the improved confinement in DT starts right at the top of the pedestal, as shown in figure 4, where the ion thermal pressure is compared for DD and DT. This trend has been also found in experimental isotope scans with hydrogen and deuterium in JT-60U [21], indicating a possible common explanation for multi-ion and single ion isotope effects.
Table 2. Comparison between the thermal energy content obtained in DD and DT for simulation with and without $E \times B$ flow shear stabilization.

\begin{tabular}{ll}
\hline Case & Wth (MJ) \\
\hline DT with $E \times B$ & 8.8 \\
DD with $E \times B$ & 6.9 \\
DT without $E \times B$ & 5.4 \\
DD without $E \times B$ & 5.6 \\
\hline
\end{tabular}

A simple explanation can be derived taking into account that turbulent eddies can be quenched by the background $E \times B$ flow shear. This holds at least for microturbulence driven by ion temperature gradients (ITGS), referred to as the ITG mode [22], which is responsible for the heat transport in the vast majority of the present day tokamaks. The $E \times B$ flow shear is expected to be independent of the mass, $\gamma_{E \times B} \sim E_{\mathrm{r}} / L$ with $E_{\mathrm{r}}$ the radial electric field and $L$ a suitable length, whereas the ITG growth rate scales as $\gamma_{\mathrm{ITG}} \sim v_{\mathrm{th}, \mathrm{i}} / L$ with $v_{\mathrm{th}, \mathrm{i}}$ the ion thermal velocity. Therefore, the ratio $\gamma_{E \times B} / \gamma_{\mathrm{ITG}}$, a measure of the impact of the external $E \times B$ flow shear on turbulence, scales as $\gamma_{E \times B} / \gamma_{\mathrm{ITG}} \sim m_{i}{ }^{1 / 2}$ indicating that the effectiveness of the $E \times B$ flow shear for quenching ITG transport increases with the mass at constant $\gamma_{E \times B}$. This possibility is verified by performing two further simulations with DT and DD without the impact of $E \times B$ flow shear. Unlike the case with $E \times B$, the plasma thermal energy content, shown in table 2, decreases with DT with respect to DD, showing the capital importance of $E \times B$ stabilization for properly accounting the impact of mass exchange. However, in strong electromagnetic turbulence, i.e. at high electron beta, the effect of $E \times B$ flow shear could be overestimated by quasi-linear models and actually the electromagnetic stabilization be responsible for most of the reduced turbulence [9]. Recent gyrokinetic simulations for the ITER hybrid scenario show that a strong DT isotope effect can be obtained by the concomitant impact of $E \times B$ flow shear, electromagnetic effects and zonal flows [23] leading to otherwise heat fluxes reductions similar to the one obtained with TGLF for which just the impact of $E \times B$ flow shear is the main cause of a strong isotope effect. Therefore, detailed heat fluxes comparisons between TGLF and non-linear gyrokinetic simulations are required for properly address the isotope effect. 

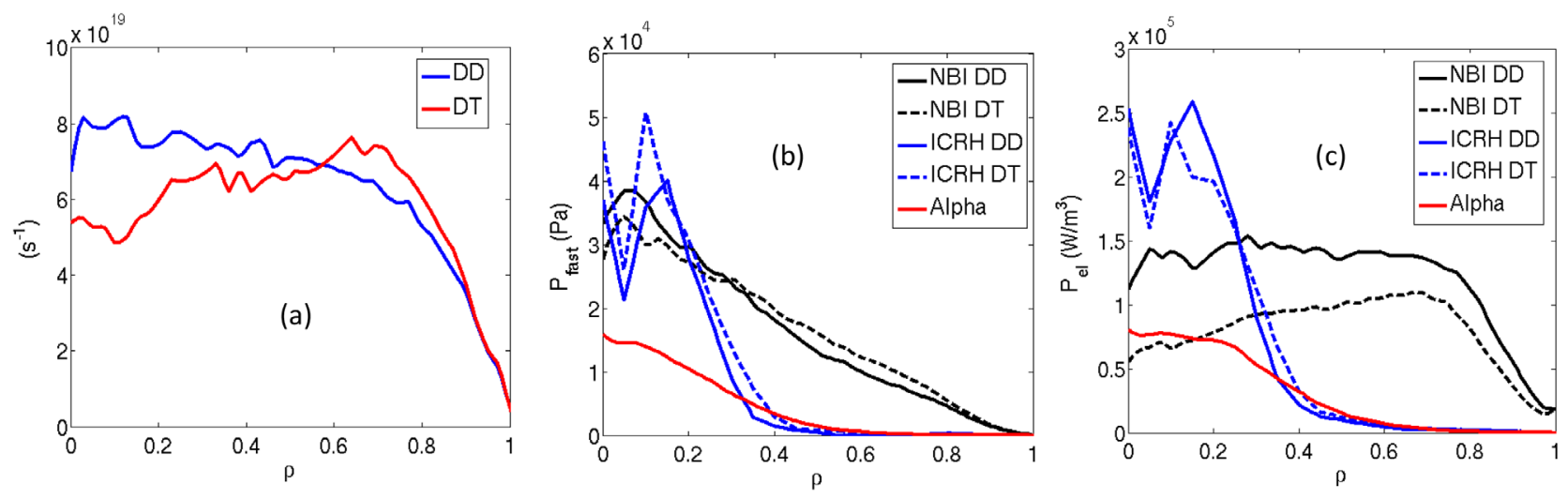

Figure 5. Comparison between the NBI fuelling in DD and DT including T-beams (a) ICRH, NBI and alpha fast ion pressure in DD and DT (b) NBI, ICRH and alpha electron power density heating in DD and DT (c).

Finally, a self-consistent simulation including alpha heating effects (thermal) calculated with code SPOT and heating using T-NBI beams has been performed with the aim of verifying their impact on the final fusion energy performance. Here, the fast ions generated by the fusion reactions have been also included in TGLF as extra specie. This extra comparison highlights some important differences between DD and DT as shown in figure 5. The T-Beam penetration becomes weaker for DT due to the higher mass of the T beams leading to a NBI core heating and fueling deficit, however additional effects can counteract it such as the contribution of the fast alphas to the fast ions pressure, which is not negligible and can have a significant impact on the ion heat transport suppression. The electron alpha heating power is comparable to the NBI in the inner core and can at least partially overcome the loss of central heating in DT. Therefore, the final fusion power including all the elements, $P_{\text {fus }}=15.5 \mathrm{MW}$ does not significantly change with respect the one previously obtained.

\section{Conclusions}

A modeling activity in support of the future JET-DT campaign has been started with the aim of highlighting the fundamental differences of plasmas composed by DT mixtures and also for guiding experimental campaigns towards maximizing fusion power. For that purpose, high beta domain has been chosen due to its low power degradation obtained in low gas conditions, something beneficial for maximizing the fusion power at high input power.

An optimum plasma operational point, in terms of electron density, has been found due to the good penetration of the NBI power at lower average density. However, enough central heating with ICRH should be guaranteed in order to overcome possible deviations from the expected density leading to a sudden drop of the NBI heating efficiency.

The necessity of low density is also a consequence of the fact that a strong isotope effect is expected in plasmas where $E \times B$ flow shear stabilization is important and therefore the plasma torque should be maximized. The impact of $E \times B$ is double, in the core, where turbulence is almost suppressed in DT and next to the top pedestal where there is a clear improvement in confinement. On the other hand, the pedestal shoulder could be unaffected as already shown in single isotope experiments from H to D in JT-60U.

Regarding alpha power effects, assuming that the whole JET heating power is available, the fusion power generated should have a noticeable impact on the fast ion fraction and pressure gradients. This will allow the analysis of toroidal Alfvén eigenmodes (TAE) stability and the impact on turbulence reduction. An important contribution to the electron heating is also observed from the simulations. This opens up the possibility of analyzing some key physics expected in ITER, such as the impact of alphas fast ion pressure and electron heating on tungsten impurity transport, already at JET.

These results are the initial step in the challenging task of predicting DT plasmas. Additional analyses and simulations including both integrated and first-principle modelling are required to address different ion particle transport for $\mathrm{D}$ and $\mathrm{T}$, impurity transport, fast ions interplay with MHD or power exhaust. All these physical ingredients are essential in order to properly extrapolate present-day plasmas to DT and future work and priorities will be established in this direction.

\section{Acknowledgments}

This work has been carried out within the framework of the EUROfusion Consortium and has received funding from the Euratom research and training programme 2014-2018 under grant agreement No 633053. The views and opinions expressed herein do not necessarily reflect those of the European Commission

\section{References}

[1] Paméla J et al 2007 Fusion Eng. Des. 87590

[2] Gormezano C et al 1998 Phys. Rev. Lett. 805544

[3] Budny R V et al 2016 Nucl. Fusion 56056002

[4] Weisen H et al 2014 AIP Conf. Proc. 161277

[5] Challis C D et al 2015 Nucl. Fusion 55053031

[6] Garcia J and Giruzzi G 2013 Nucl. Fusion 53043023

[7] ITER Physics Basis 1999 Nucl. Fusion 392175

[8] Citrin J et al 2013 Phys. Rev. Lett. 111155001

[9] Garcia J et al 2015 Nucl. Fusion 55053007

[10] Artaud J F et al 2010 Nucl. Fusion 50043001 
[11] Huysmans G T A et al 1991 CP90 Conf. on Computational Physics (Word Scientific) p 371

[12] Schneider M et al 2011 Nucl. Fusion 51063019

[13] Eriksson L-G, Hellsten T and Willén U 1993 Nucl. Fusion 331037

[14] Staebler G M et al 2005 Phys. Plasmas 12102508

[15] Cordey J G 2003 Nucl. Fusion 43 670-4

[16] Garcia J et al 2014 Nucl. Fusion 54093010

[17] Bosch H-S and Hale G M 1992 Nucl. Fusion 32611

[18] Goldston R J et al 1981 J. Comput. Phys. 43 61-78
[19] Mantsinen M J et al 2015 42nd EPS Conf. on Plasma Physics (Lisbon, Portugal, 22-26 June 2015) http://ocs.ciemat.es/ EPS2015PAP/pdf/P2.171.pdf

[20] Gallart D et al 2016 43nd EPS Conf. on Plasma Physics (Leuven, Belgium, 4-8 July 2016) P2.003

[21] Urano H et al 2012 24th Fusion Energy Conf. (San Diego, CA, USA, 8-13 October 2012

[22] Romanelli F 1989 Phys. Fluids B 11018

[23] Garcia J et al 2016 Nucl. Fusion submitted

[24] Romanelli F et al 2014 Proc. of 25th IAEA FEC (St Petersburg, Russia) 